\title{
Hybrid Regularization Algorithm for Efficient Image Deblurring
}

\author{
Pooja S, Mallikarjunaswamy S, Sharmila N.
}

\begin{abstract}
Image deblurring is a challenging illposed problem with widespread applications. Most existing deblurring methods make use of image priors or priors on the PSF to achieve accurate results. The performance of these methods depends on various factors such as the presence of well-lit conditions in the case of dark image priors and in case of statistical image priors the assumption the image follows a certain distribution might not be fully accurate. This holds for statistical priors used on the blur kernel as well. The aim of this paper is to propose a novel image deblurring method which can be readily extended to various applications such that it effectively deblurs the image irrespective of the various factors affecting its capture. A hybrid regularization method is proposed which uses a TV regularization framework with varying sparsity inducing priors. The edges of the image are accurately recovered due to the $T V$ regularization. The sparsity prior is implemented through a dictionary such that varying weights of sparsity is induced based on the different image regions. This helps in smoothing the unwanted artifacts generated due to blur in the uniform regions of the image.
\end{abstract}

Keywords: Image Priors, Maximum A Posteriori Estimation, Regularization

\section{INTRODUCTION}

The widespread applications of digital images such as in astronomical imaging, medical imaging law and order maintenance, remote sensing etc. has led to greater interest in improving the existing image deblurring methods. This has attracted wide research from various researches around the world. Image deblurring is the process of recovering the true sharp image from a degraded image which is both blurry and noisy. The image blurring operation is most effectually represented as a convolution operation, whereas noise which is additive in nature is represented by the summation operation. The general mathematical expression for the image deblurring model is given in the equation below [1]

$$
\boldsymbol{g}=\boldsymbol{H} * \boldsymbol{u}+\boldsymbol{n}
$$

where $\mathbf{g}$ represents the captured degraded image, which is both blurry and noisy and $\mathbf{u}$ represents the true sharp image, both these images are represented through

Manuscript received on August 21, 2021.

Revised Manuscript received on August 24, 2021.

Manuscript published on August 30, 2021.

* Correspondence Author

Pooja S.*, Assistant Professor, Department of ECE, K.S. Institute of Technology, Bengaluru, India. Email: poojas@ksit.edu.in

Mallikarjunaswamy S., Assistant Professor, Department of ECE, JSS Academy of Technical Education, Bengaluru, India. Email: pruthvi.malli@gmail.com

Sharmila N., Assistant Professor, Department of ECE, RNSIT, Bengaluru, India.

(C) The Authors. Published by Blue Eyes Intelligence Engineering and Sciences Publication (BEIESP). This is an open access article under the CC BY-NC-ND license (http://creativecommons.org/licenses/by-nc-nd/4.0/) matrices such that $\mathbf{g}$ and $\mathbf{u} \in \mathrm{R}^{\mathrm{N}}(\mathrm{N}=\mathrm{m} \times \mathrm{n})$. $\mathbf{H}$ denotes the point spread function responsible for the blurring in the image, $*$ denotes the mathematical convolution operation, $\mathbf{n}$ is the noise, where $\mathbf{n} \in \mathrm{R}^{\mathrm{N}}$ and it is assumed to be white Gaussian noise with variance $\sigma^{2}$. The problem statement in equation (1) is extremely illposed due to the presence of multiple not unique solutions to the true sharp image $\mathbf{u}$ and the blur kernel $\mathbf{H}$. Deblurring is the process of effectively recovering the blur function $\mathbf{H}$ and subsequently acquiring the true sharp image $\mathbf{u}$ from it.

Blurring is caused due to various factors such as relative motion between the objects in the scene and the camera, weather conditions such as rain or smog another contributing factor for the quality of image is the light intensity of the scene capture. If an image is captured in dim light conditions the effect of blurring tends to be more compared to an image of the same scene captured in well-lit conditions. Similarly, few other factors which cause blurring are camera hardware specifications, imperfections in the lens, out of focus blur caused due to the object being out of range from the optical axis of the camera lens etc. Blurring is inevitable in digital cameras and requires image deblurring techniques to restore the captured images which are vital in applications such as medical imaging, remote sensing, law and order maintenance through surveillance cameras etc.

\section{RELATED WORK}

Blind Deconvolution for image restoration has led to many publications starting from a simple iterative algorithm of assuming a known PSF to various non iterative algorithms. Iterative algorithms such as Expectation-maximization algorithm and Maximum Posteriori Estimation. The Maximum Posteriori Estimation methods have proved improved PSF estimation. Numerous blind image deblurring algorithms have been proposed in recent times. Most of these methods make use of image priors to improve the performance of the MAP estimation technique. Khongkraphan et al. [1] propose a smoothing prior to reduce the blur artifacts in the image. Zhou et al [2] propose the use of joint extreme channel and L0 priors on the entire image. Zhao et al. [3] use gradient distribution priors to derive efficient deblurring results from the MAP method. Gu et al. [4] use both and local and non-local priors to denoise the image. Qin et al [5] use an extremums prior based in the image intensity to improve deblurred image quality. Zhang et. al [6] proposes iteration-wise assumption of priors in an alternating minimization algorithm.

Published By:

Blue Eyes Intelligence Engineering and Sciences Publication

(C) Copyright: All rights reserved.

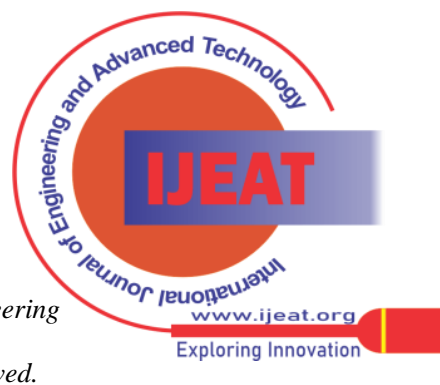


They propose a training algorithm to learn the best $L_{p}$ prior for each iteration. Iterative procedures of various methods have been proposed before. But here a prior suited for each iteration is derived from the training algorithm.

A probabilistic MAP Maximum A Posteriori Estimation model is used to simplify the ill-posed problem. Perrone and Favaro [7] propose an Anisotropic version of Total Variation Blind Deconvolution with a sum of $L_{1}$ norms of gradient of $u$ used as prior. Here the stress being on an inferred sum of different priors chosen. The prior used depends on the gradient of the image.

Xue, et. al [8] propose a statistical and non-Bayesian algorithm SURE (Stein's unbiased risk estimate). Optimization is found to be of atmost importance in an iterative procedure. It increases the accuracy PSF estimation. Variations of Augmented Lagrangian Methods are used for optimization of image and PSF estimates. Whereas Javier Portilla, Antonio Tristán-Vega et. al [9] propose a sparsity-enforcing term which acts as a prior, on a Bayesian view. The prior is derived by assuming a varied form of $l$ norm. The prior fits a standard MAP estimation model. It implements a regularizing algorithm converting most co-efficients of the image to be zero. Pan, et. al [10] propose a single convolution filter to approximate the spatially heterogeneous median filter. The median filter which is applied on each and every pixel specifically is varied to perform in a single convolution operation. A variation of the deconvolution algorithm is proposed for image enhancement. Micheal Bromberger et. Al [11] propose an FPGA implementation of the straightforward Richardson-Lucy deconvolution algorithm. The hardware implementation yields better deconvolution results, than the software implementation of the same algorithm. These methods and the merits of these algorithms and their implementation along with the drawbacks of these methods are detailed in our previous work [12]. Filip Sroubek and Peyman Milanfar [13] propose a variation in the iterative algorithm by using multiple input images for observation (MCBD). The use of multiple images derived better results compared to algorithms using a single input image or Single Channel Blind Deconvolution (SCBD). But it is also understood from the above papers that for accurate PSF estimation assumption of accurate priors plays a vital role. Hence the accuracy of an algorithm depends largely on the priors assumed. Keeping this in mind, though the SCBD problem is extremely ill-posed it is chosen as the problem statement for solving the image restoration problem. This is mainly due to the vast scope of improvement and the flexibility in employing various methods that is not possible in an MCBD problem statement.

\section{REGULARIZATION}

Various methods for image deblurring have been proposed over the years. Initially, a wide variety of filters have been proposed two remove the effects of noise in the image. In order to mitigate the effects of both noise and blur a filter alone is not adequate. This has led to the use of various regularization techniques. One popular regularization technique used for image deblurring is the Lp norm regularization. Varying the value of $p$ here varies the regularization method and leads to different results with respect to the optimum value selection. Each of these methods and their possible results have been extensively studied in our previous work [12]. Each of these various techniques provide valuable insight into the performance of an image deblurring algorithm using the Maximum A Posteriori (MAP) estimation technique. The Total Variation (TV) Prior has an advantage of retaining the sharp edges of the image, whereas Tikhonov and Sobolev priors blur the sharp edges of the image. The TV prior for an image $\mathrm{x}$ is given as:

$$
\Pi(x)=\underset{i}{\Sigma}\|\nabla x(i)\|
$$

The TV prior is, a non-smooth prior which requires complex convex optimization techniques to be implemented as regularization. By using a smoothened TV Regularizer, the complexity can be simplified. The smoothened TV norm is given by the equation below:

$$
\Pi(x)=\sum_{i} \sqrt{\|\nabla x(i)\|^{2}+\varepsilon^{2}}
$$

\section{PROPOSED METHOD}

The MAP model is the general frame work used to integrate the various regularization techniques in an image deblurring algorithm. The mathematical expression representing the MAP general model is represented in the equation below

$$
\{\tilde{u}, \widehat{h}\}=\arg \min \|\mathbf{h} * \mathbf{u}-\mathbf{g}\|^{2}+\lambda \rho(u)+\beta \rho(h)
$$

The popular TV regularization technique is used in the proposed image deblurring algorithm, considering its effectiveness in preserving the edges of the image. The main limitation of the TV regularization is that it leads to artifacts in the uniform regions of the image. In order to facilitate adaptive regularization which varies with respect to the spatial regions of the image we introduce a regularization parameter $\gamma$. The regularization parameter is estimated keeping two main requirements of efficient image deblurring

1) Preservation of the true edges of the image

2) Suppression of artifacts caused due to blur

The regularization parameter is a variable, which depends on the local image features. This leads to a spatially adaptive regularization., the regularization parameter $\gamma$ enforces additional weight to the regularization term to ensure smoothness in the uniform regions and avoid the artifacts caused due to blurring. The proposed image deblurring model based on the TV regularization framework, along with an adaptive sparsity prior is shown in the equation below

$$
\tilde{u}, \widehat{h}\}=\arg \min \|\mathrm{h} * \mathrm{u}-\mathrm{g}\|^{2}+\gamma\left\|\mathrm{S}^{\mathrm{B}} \mathrm{u}\right\|+\beta \rho(\mathrm{h})
$$

The sparsity prior is used in the form of regularization. The regularization is performed through the use of a dictionary which is predefined such that it is orthogonal. Due to its orthogonal nature the coefficients can also be represented as $\boldsymbol{\mu}=\mathbf{S}^{\mathbf{B}} \mathbf{u}$. Along with the knowledge of the salient edges preserved through the TV regularization, the sparsity prior acts as an image smoothening prior.

Published By: Blue Eyes Intelligence Engineering and Sciences Publication

(C) Copyright: All rights reserved.

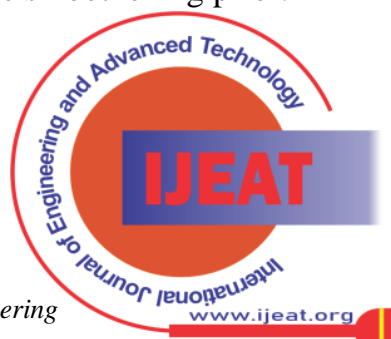


The parameter $\gamma$ ensures only the uniform regions of the image is smoothened. The varying weights of $\gamma$ smoothens the ringing and step artifacts caused due to blur.

Apart from the selection of the right prior the success of the proposed image deblurring algorithm depends on the correct implementation technique. Theoretical findings on several blind deconvolution algorithms generated contrasting views on why these approaches worked. On the one hand, one could observe experimentally that alternating energy minimization algorithms converge to the desired solution. On the other

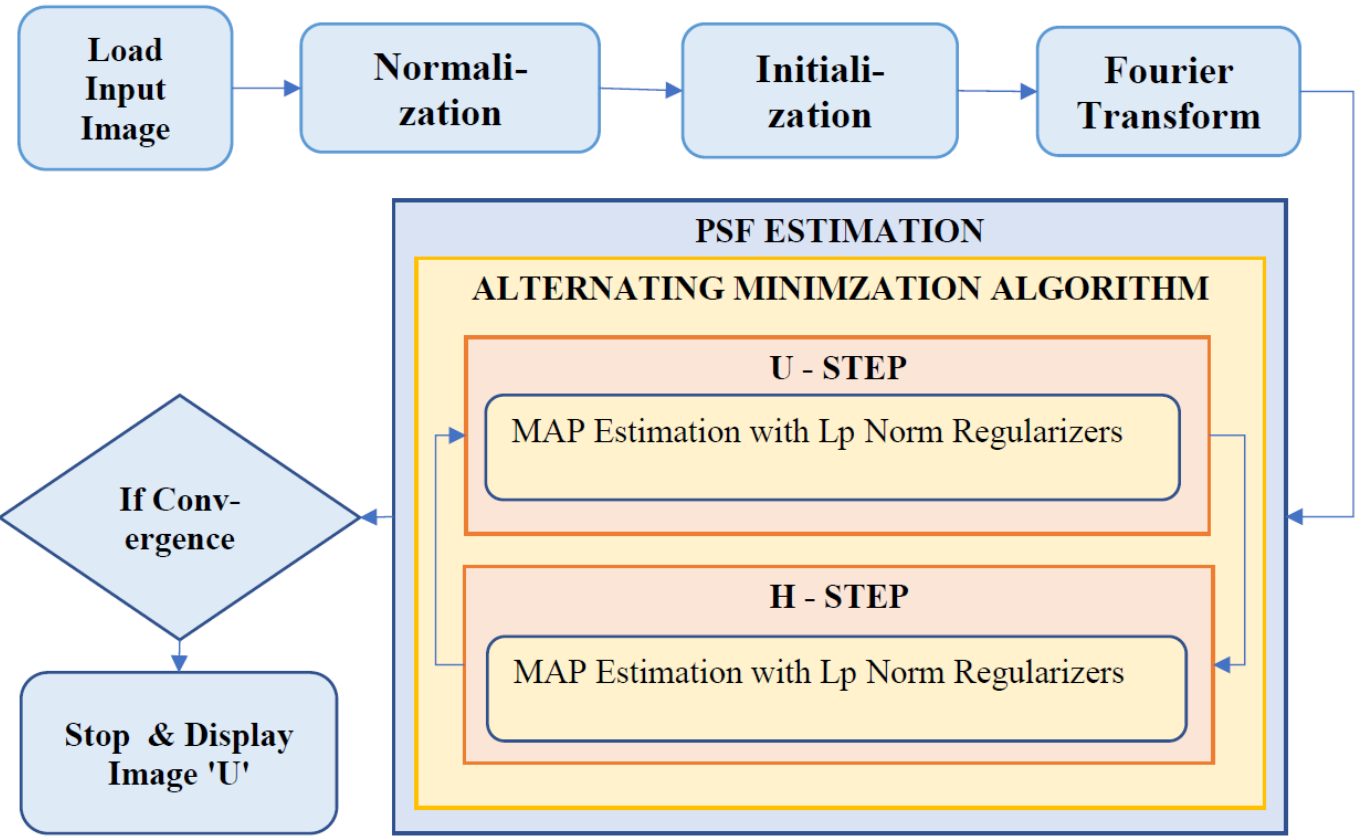

Figure 1: The sequence of different steps in the proposed image deblurring algorithm is depicted

hand, it has been shown that such alternating minimization algorithms should fail to converge. An in-depth analysis of Total Variation Blind Deconvolution is required to better understand this incongruence. The reason for deriving such varied results using the same algorithm i.e. Total Variation Blind Deconvolution (TVBD) is due to the variation in implementing the TVBD algorithm. Its specific implementation by Chan et.al [7] converges to a desired solution, even when starting at the no-blur solution.

The effectiveness of the TV regularization lies in the method of implementation. Here in order to efficiently utilize the TV regularization, the constraints are initially removed during the gradient descent step and then the constraints are sequentially applied. In a Total Variation Blind Deconvolution model, the cost functional is not convex. Hence this completely changes the behavior of the algorithm ensuring convergence.

\section{HYBRID REGULARIZATION ALGORITHM}

The proposed blind image deblurring algorithm make use of the Maximum A Posteriori Estimation mathematical model depicted in equation (5). The main contribution of the algorithm is in its method of implementation. The optimization problem is solved using the alternating minimization algorithm, which alternates between the estimation of the sharp image and the true blur kernel, shown in figure1. The minimum of a real-valued function $\mathrm{f}(\mathrm{x})$ can be found by a method minimum optimization. It is a technique of estimating the absolute or the global minima of a function.

$\mathrm{s}$ are initially solved without the constraints. This avoids the selection of local minima. The constraints are completely avoided in the H-step and are introduced in the U-step only when the weight of the regularization parameter $\gamma$ is considerably large. This ensures the implementation of constraints only in the uniform regions of the image, which in turn further smoothens the uniform regions of the image by smoothening any artifacts such as ringing or staircase artifacts formed while the sharp edges of the image are not smoothened.

\section{Algorithm: Hybrid Regularization Algorithm}

Input: Captured Image G

Estimate: True Blur Kernel $\mathbf{H}$ \& sharp image $\boldsymbol{u}$ Process:

1. Initialize: $\max \mathrm{H}$ size $=12 \mathrm{x} 12, u=\mathrm{g}$

2. Analyze: image regions through its spectral components

3. Categorize: the image into different regions based on step 2

4. Apply: TV regularization and sparsity through the mathematical model of Eq. 5

5. Implement: Alternating Minimization Algorithm

6. Estimate $\mathrm{H}$

7. Use $\mathrm{H}$ update to estimate $u$

8. Use u estimate to update $\mathrm{H}$

9. Repeat until convergence

\section{Output: $u$}

The algorithm is implemented in the spectral domain, this reduces the computational complexity of the algorithm. The illposed problem is solved by using two different regularizers the TV norm and sparse priors.

Published By:

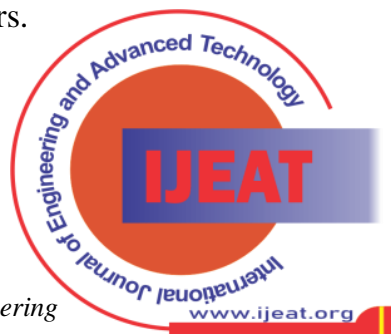


A TV regularizer would suffice to convert the NP-hard into a problem which is executable in finite time. The use of a second sparsity prior is to ensure the smoothening of the staircase and ringing artifacts in the deblurred result caused due to regularization using the TV norm. Fixing the $\mathrm{P}$ value to 1 in an Lp norm regularization technique reduces the problem to a TV regularization and fixing the $\mathrm{P}$ value to 0 leads to L0 regularization. Some methods propose the use variable $\mathrm{P}$ value which helps in achieving the smoothing of unwanted artifacts. But this method is not as effective as a TV norm in retaining the sharp edges of the image. Hence the TV norm is used to ensure the preservation of the sharp edges of the image and a separate sparsity inducing prior is to smoothen the artifacts. The input image is normalized and the size of the blur kernel is initialized. This input image is further converted into the frequency domain through the use of Fourier Transform. The true PSF is estimated through an alternating minimization algorithm which alternates minimizing the energy between the sharp image and the true
PSF. The u-step estimates the sharp image while the h-step uses the u estimate from the u-step to estimate the true blur kernel. This repeats until the algorithm reaches the convergence criteria. The sparsity driven image regularization is implemented through a dictionary represented by the second term in the RHS of equation (5). The dictionary aids in implementing different levels of sparsity in different image regions, categorized through their spectral information. The regularization parameter $\gamma$ further ensures that the sharp regions of the image remain untouched whereas the uniform regions of the image. The use of specific weights of sparsity in each image region leads to improved deblurred results. The use of varying weights is proportional to varying $\mathrm{P}$ values in the $\mathrm{Lp}$ norm but has an added advantage of sharp edges preserved in the estimated sharp image. The $\mathrm{P}$ value is a very important parameter in the $\mathrm{Lp}$ norm the dictionary enforces sparsity depending upon the value of $\gamma$ which is dependent on the image region estimation based on the spectral components of the image.

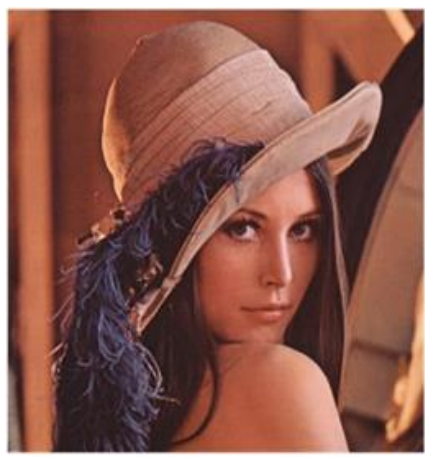

(a)

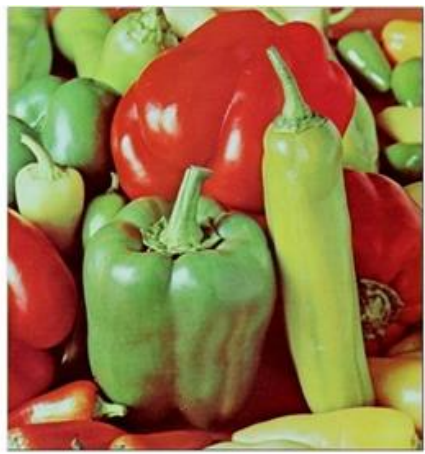

(b)

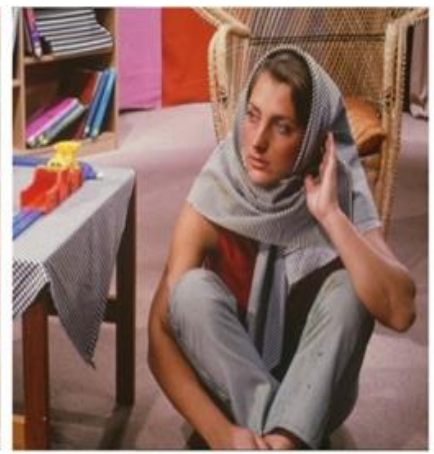

(c)

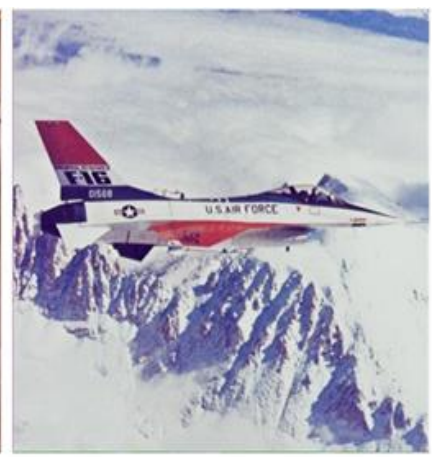

(d)

Figure 2: Four different color test images of size $256 \times 256 \times 3$ : (a) Lena, (b) Peppers, (c) Barbara, and (d) Airplane

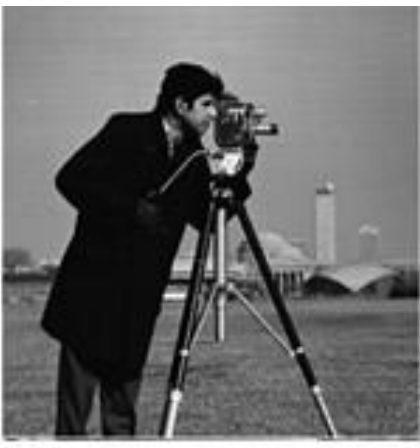

(a)

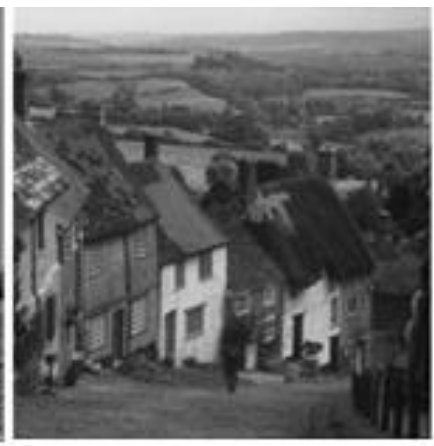

(b)

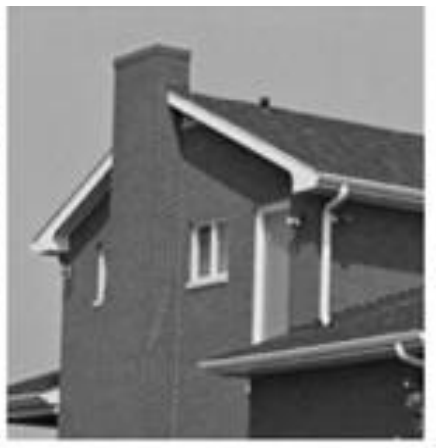

(c)

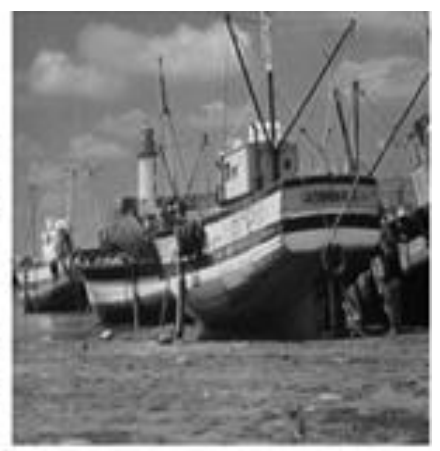

(d)

Figure 3: Four grayscale test images of size $256 \times 256$ (a) Lena, (b) Peppers, (c) Barbara, and (d) Airplane

\section{RESULTS AND DISCUSSIONS}

The proposed hybrid regularization algorithm is implemented in MatLab, using an intel i5 8265U Processor @ $1.80 \mathrm{GHz}$ with an 8GB SSD and a 64-bit based processor with a 64-bit Windows OS. The performance of the hybrid regularization algorithm is experimentally tested using both color images and grayscale images. The four-color images and the four-grayscale images used as test images for the experimental study is shown in figure 2 and figure 3 above. The PSNR values of the deblurred images using different methods are tabulated in Table 1 , the deblurred results using the proposed hybrid regularization algorithm shows improved image quality compared to recent state-of-the art image deblurring algorithms. Similarly, the SSIM results of the deblurred images using different methods are tabulated in Table 2. The quality of the deblurred image using the proposed hybrid

Blue Eyes Intelligence Engineering and Sciences Publication

(C) Copyright: All rights reserved.

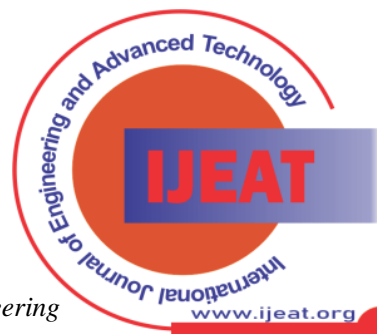


Table- I: PSNR comparisons of various image restoration methods on test images for restoring blurred images corrupted by different variance $\left(\sigma^{2}\right)$ values of Gaussian blur

\begin{tabular}{|c|c|c|c|c|c|c|c|c|}
\hline$\sigma^{2}$ Methods & Lena & Cameraman & Barbara & Boats & Hill & House & Man & Peppers \\
\hline 2.5 TV & 22.942 & 23.981 & 23.973 & 22.081 & 24.364 & 27.253 & 22.98 & 24.575 \\
\hline Edge Priors & 23.721 & 24.526 & 24.413 & 22.855 & 25.13 & 28.468 & 24.102 & 25.714 \\
\hline Sparsity & 25.043 & 25.505 & 24.964 & 23.946 & 26.357 & 20.022 & 25.641 & 27.144 \\
\hline L1 & 25.554 & 25.975 & 25.236 & 24.482 & 26.83 & 30.547 & 26.133 & 27.724 \\
\hline L0 & 26.252 & 26.608 & 25.614 & 25.313 & 27.556 & 31.194 & 26.968 & 28.564 \\
\hline Proposed Method & 26.423 & 26.954 & 25.826 & 25.597 & 27.743 & 31.321 & 27.215 & 28.884 \\
\hline 3.5 TV & 22.71 & 23.342 & 23.774 & 21.605 & 24.053 & 26.947 & 22.544 & 23.613 \\
\hline Edge Priors & 23.453 & 23.685 & 24.032 & 22.156 & 24.87 & 27.612 & 23.205 & 24.422 \\
\hline Sparsity & 24.116 & 24.122 & 24.463 & 22.924 & 25.458 & 28.78 & 24.503 & 25.694 \\
\hline L1 & 24.465 & 24.412 & 24.71 & 23.284 & 25.866 & 28.207 & 24.94 & 25.921 \\
\hline L0 & 25.072 & 24.743 & 24.915 & 23.743 & 26.246 & 29.857 & 25.715 & 26.577 \\
\hline Proposed Method & 25.252 & 25.103 & 25.124 & 24.125 & 26.583 & 30.145 & 25.904 & 26.826 \\
\hline 4.5 TV & 22.291 & 22.562 & 23.363 & 20.792 & 23.523 & 26.582 & 22.243 & 22.475 \\
\hline Edge Priors & 22.643 & 22.752 & 23.64 & 21.135 & 23.764 & 26.865 & 22.602 & 22.828 \\
\hline Sparsity & 23.142 & 23.085 & 23.863 & 21.655 & 24.23 & 27.383 & 23.372 & 23.486 \\
\hline L1 & 23.324 & 23.265 & 24.004 & 21.88 & 24.362 & 27.6 & 23.613 & 23.756 \\
\hline L0 & 23.553 & 23.482 & 24.176 & 22.268 & 24.723 & 27.935 & 23.967 & 24.173 \\
\hline Proposed Method & 23.782 & 23.764 & 24.371 & 22.472 & 24.99 & 28.102 & 24.114 & 24.365 \\
\hline T.5 TV & 21.828 & 21.922 & 22.761 & 20.077 & 22.902 & 25.635 & 21.683 & 21.046 \\
\hline Edge Priors & 22.016 & 22.063 & 22.904 & 20.284 & 23.105 & 25.823 & 21.875 & 21.31 \\
\hline Sparsity & 22.324 & 22.357 & 23.175 & 20.56 & 23.177 & 26.079 & 22.134 & 21.845 \\
\hline L1 & 22.507 & 22.504 & 23.355 & 20.772 & 23.322 & 26.201 & 22.33 & 22.283 \\
\hline L0 & 22.702 & 22.714 & 23.583 & 21.164 & 23.554 & 26.426 & 22.587 & 22.694 \\
\hline Proposed Method & 22.884 & 22.875 & 23.706 & 21.342 & 23.725 & 26.658 & 22.775 & 22.902 \\
\hline
\end{tabular}

Table-II: SSIM comparisons of various image restoration methods on test images for restoring blurred images corrupted by different variance $\left(\sigma^{2}\right)$ values of Gaussian blur

\begin{tabular}{|c|c|c|c|c|c|r|r|r|}
\hline$\sigma^{2}$ Methods & Peppers & Airplane & Barbara & Lena & Hill & House & Man & Peppers \\
\hline 1.5 & 0.801 & 0.8324 & 0.8049 & \multirow{2}{*}{0.7972} & \multirow{2}{*}{0.7999} & \multirow{2}{*}{0.8588} & \multirow{2}{*}{0.7936} & 0.8753 \\
\hline TV & 0.8384 & 0.8478 & 0.8224 & 0.8355 & 0.839 & 0.8793 & 0.8477 & 0.899 \\
\hline Edge Priors & 0.855 & 0.8565 & 0.8452 & 0.8572 & 0.8583 & 0.8937 & 0.8723 & 0.9119 \\
\hline Sparsity & 0.8662 & 0.8637 & 0.8503 & 0.8715 & 0.8764 & 0.9068 & 0.8836 & 0.9198 \\
\hline L1 & 0.8859 & 0.8667 & 0.8743 & 0.8891 & 0.9082 & 0.9271 & 0.9015 & 0.9301 \\
\hline L0 & 0.889 & 0.8702 & 0.8775 & 0.8965 & 0.9117 & 0.9305 & 0.9062 & 0.9342 \\
\hline Proposed Method & 0.7931 & 0.8203 & 0.7955 & 0.776 & 0.781 & 0.8525 & 0.7791 & 0.8621 \\
\hline TV & 0.8106 & 0.8257 & 0.8068 & 0.7924 & 0.8027 & 0.8636 & 0.8065 & 0.883 \\
\hline Edge Priors & 0.8278 & 0.8313 & 0.8212 & 0.8177 & 0.8214 & 0.8771 & 0.8331 & 0.9053 \\
\hline Sparsity & 0.8412 & 0.834 & 0.8355 & 0.8316 & 0.8352 & 0.8913 & 0.8485 & 0.9093 \\
\hline L1 & 0.8546 & 0.8374 & 0.8477 & 0.8451 & 0.8486 & 0.9031 & 0.8632 & 0.9138 \\
\hline L0 & 0.8613 & 0.8433 & 0.8525 & 0.85 & 0.8559 & 0.909 & 0.8675 & 0.916 \\
\hline Proposed Method & 0.7746 & 0.7894 & 0.7803 & 0.7306 & 0.7455 & 0.8453 & 0.7502 & 0.8464 \\
\hline TV & 0.7865 & 0.7928 & 0.7846 & 0.7422 & 0.7528 & 0.8517 & 0.7638 & 0.8503 \\
\hline Edge Priors & 0.8004 & 0.7955 & 0.7904 & 0.7606 & 0.7682 & 0.8596 & 0.7794 & 0.8563 \\
\hline Sparsity & 0.805 & 0.7968 & 0.7971 & 0.774 & 0.7773 & 0.8621 & 0.7917 & 0.8592 \\
\hline L1 & 0.813 & 0.8054 & 0.8142 & 0.7936 & 0.7938 & 0.8651 & 0.8105 & 0.8648 \\
\hline L0 & 0.8164 & 0.811 & 0.8225 & 0.8014 & 0.8023 & 0.8714 & 0.8183 & 0.8705 \\
\hline Proposed Method & 0.7466 & 0.7407 & 0.7493 & 0.6952 & 0.7009 & 0.8278 & 0.7113 & 0.813 \\
\hline TV & 0.7463 & 0.732 \\
\hline Edge Priors & 0.759 & 0.751 & 0.7607 & 0.707 & 0.7132 & 0.832 & 0.7307 & 0.8305 \\
\hline Sparsity & 0.7737 & 0.7758 & 0.7724 & 0.7212 & 0.7412 & 0.8405 & 0.7458 & 0.8411 \\
\hline L1 & 0.781 & 0.78 & 0.7809 & 0.7406 & 0.76 & 0.8444 & 0.7578 & 0.8456 \\
\hline L0 & 0.7903 & 0.7866 & 0.7971 & 0.7764 & 0.7768 & 0.8513 & 0.7842 & 0.8507 \\
\hline Proposed Method & 0.795 & 0.7906 & 0.8002 & 0.781 & 0.7815 & 0.8554 & 0.792 & 0.8551 \\
\hline
\end{tabular}

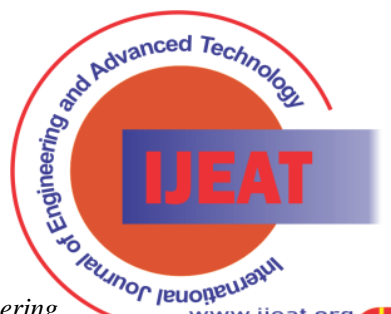




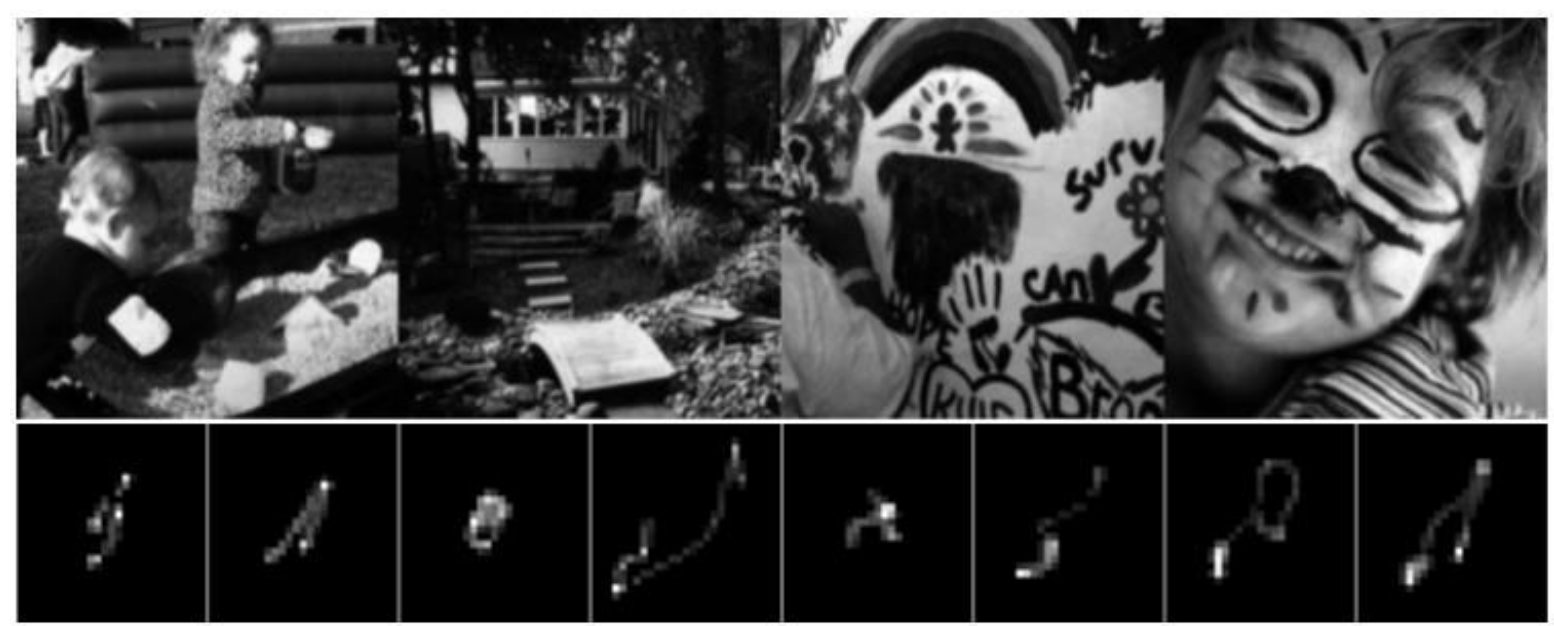

Figure 4. The four test images along with the eight PSFs $(4 \times 8=32)$ constitute the 32 images of the Levin et.al. test dataset used to assess the performance of the proposed Hybrid Regularization Algorithm in comparison with state-of-the-art image deblurring algorithms.

\section{MSE Results [1-16]}

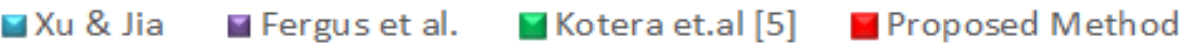

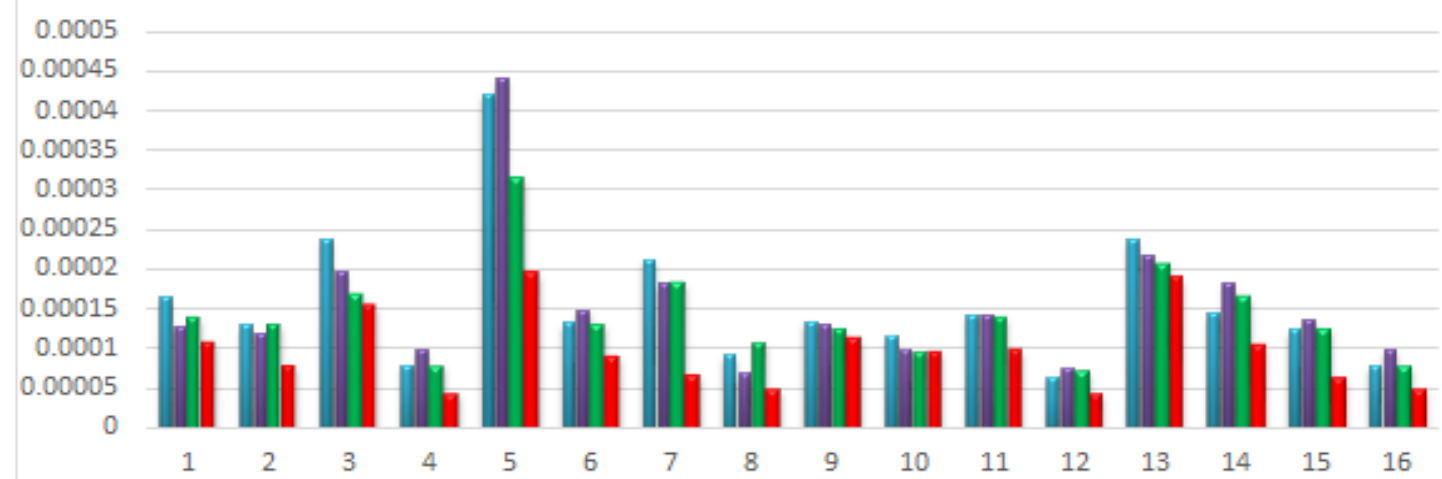

Figure 5. The performance of the proposed Hybrid Regularization Algorithm is assessed with respect to MSE and is compared with other state-of-the-art image deblurring methods, using the first 16 test images of Levin et. al. data set.

\section{MSE Results [17-32]}

口 Xu \& Jia $\square$ Fergus et al.

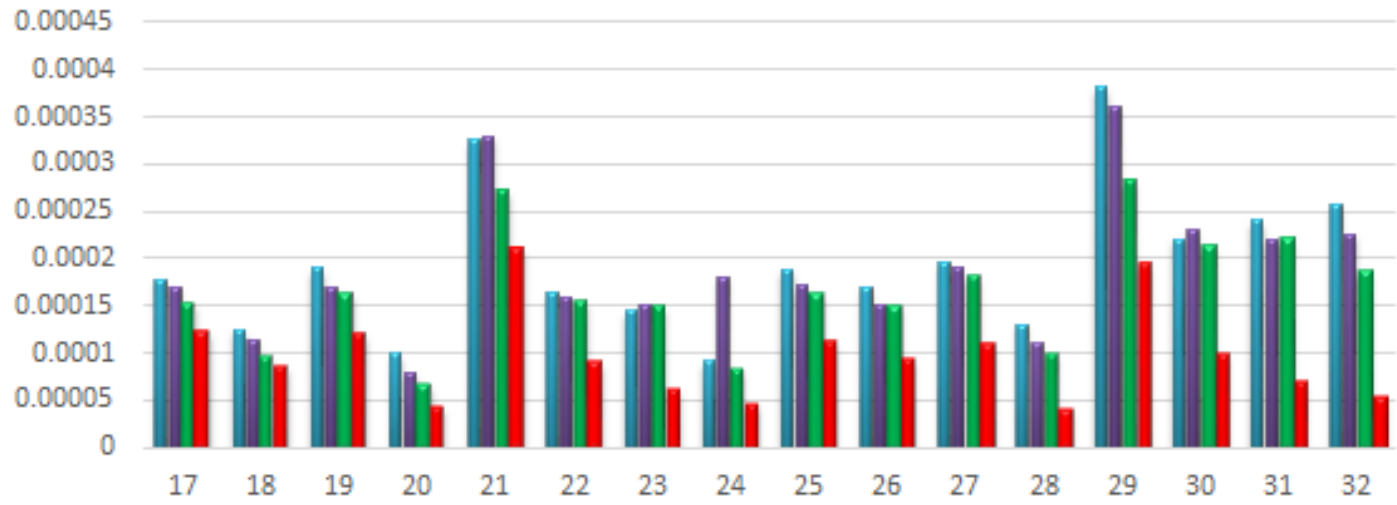

Figure 6. The performance of the proposed Hybrid Regularization Algorithm is assessed with respect to MSE and is compared with other state-of-the-art image deblurring methods, using the last 16 test images of Levin et. al. data set.

Published By:

Blue Eyes Intelligence Engineering

and Sciences Publication

(C) Copyright: All rights reserved.

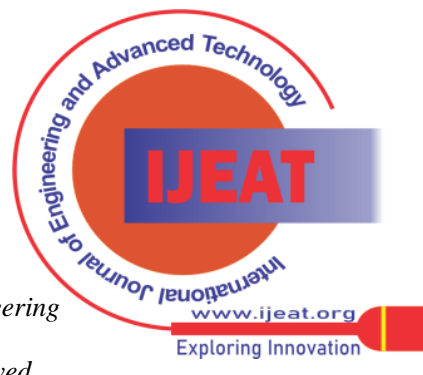




\section{RESULTS AND DISCUSSIONS}

Regularization algorithm is higher compared to recent image deblurring algorithms. The standard test data set of Levin et. al. comprises of the four grayscale images and eight blur kernels, together these form $4 \times 8=36$ test images, shown in Figure 3. These 36 images are used to asses the performance of the proposed hybrid regularization algorithm. The error ratios of the deblurred images are calculated and their MSE (Mean Square Error) values are depicted in the graphs shown in Figure 4 and 5 . It is seen that the Mean Square Error values of the deblurred images using the proposed method is lower compared to popular image deblurring algorithms, this shows improved performance of the proposed algorithm using three performance analysis metrics i.e., the PSNR, SSIM and MSE

\section{CONCLUSION}

The use a common prior over the entire image though is effective in some regions of the image such as the sharp edges of the image they fail to recover the true image value in other image regions such as the uniform regions of the image.

The Hybrid Regularization which uses two different regularization techniques ensures not only the effective preservation of the sharp edges but also smoothens the uniform regions of the image thus smoothing any unwanted staircase or ringing artifacts generated in the smooth regions, which leads to improved image deblurring performance compared to using only a single image prior over the entire image.

\section{ACKNOWLEDGMENT}

The authors would like to thank KS Institute of Technology, Bangalore, JSS Acadecmy of Technical Education, Bengaluru, Visvesvaraya Technological University (VTU), Belagavi and Vision Group on Science and Technology (VGST) Karnataka Fund for Infrastructure strengthening in Science \& Technology Level -2 for all the support and encouragement provided by them to take up this research work and publish this paper.

\section{REFERENCES}

1. Khongkraphan, K., Phonon, A. and Nuiphom, S., 2021. An Efficient Blind Image Deblurring Using a Smoothing Function. Applied Computational Intelligence and Soft Computing, 2021.

2. Zhou, K., Zhuang, P., Xiong, J., Zhao, J. and Du, M., 2020, October. Blind Image Deblurring With Joint Extreme Channels And L 0-Regularized Intensity And Gradient Priors. In 2020 IEEE International Conference on Image Processing (ICIP) (pp. 873-877). IEEE.

3. Zhao, H., Yang, H., Su, H. and Zheng, S., 2020. Natural image Deblurring based on ringing artifacts removal via knowledge-driven gradient distribution priors. IEEE Access, 8, pp.129975-129991.

4. Gu, S., Timofte, R. and Van Gool, L., 2018, August. Integrating local and non-local denoiser priors for image restoration. In 2018 24th International Conference on Pattern Recognition (ICPR) (pp 2923-2928). IEEE.

5. Qin, Z., Wu, B. and Li, M., 2018, February. Text Image Deblurring via Intensity Extremums Prior. In International Conference on Multimedia Modeling (pp. 505-517). Springer, Cham.

6. Zhang, H., Dong, Y. and Fan, Q., 2017. Wavelet frame based Poisson noise removal and image deblurring. Signal Processing, 137, pp.363-372.W.-K. Chen, Linear Networks and Systems (Book style). Belmont, CA: Wadsworth, 1993, pp. 123-135.

7. Perrone, D. and Favaro, P., 2014. Total variation blind deconvolution: The devil is in the details. In Proceedings of the IEEE Conference on Computer Vision and Pattern Recognition (pp. 2909-2916).
8. Xue, F. and Blu, T., 2014. A novel SURE-based criterion for parametric PSF estimation. IEEE Transactions on Image Processing, 24(2), pp.595-607.

9. Portilla, J., Tristan-Vega, A. and Selesnick, I.W., 2015. Efficient and robust image restoration using multiple-feature L2-relaxed sparse analysis priors. IEEE Transactions on Image Processing, 24(12), pp.5046-5059.

10. Li, L., Pan, J., Lai, W.S., Gao, C., Sang, N. and Yang, M.H., 2018 Learning a discriminative prior for blind image deblurring. In Proceedings of the IEEE Conference on Computer Vision and Pattern Recognition (pp. 6616-6625).

11. Bromberger, M., Bastian, P., Bergeest, J.P., Conrad, C., Heuveline, V., Rohr, K. and Karl, W., 2016, April. FPGA-accelerated Richardson-Lucy deconvolution for 3D image data. In 2016 IEEE 13th International Symposium on Biomedical Imaging (ISBI) (pp. 132-135). IEEE.

12. Satish, P., Srikantaswamy, M. and Ramaswamy, N.K., 2020. A Comprehensive Review of Blind Deconvolution Techniques for Image Deblurring. Traitement du Signal, 37(3).

13. Sroubek, F. and Milanfar, P., 2011. Robust multichannel blind deconvolution via fast alternating minimization. IEEE Transactions on Image processing, 21(4), pp.1687-1700.

\section{AUTHORS PROFILE}

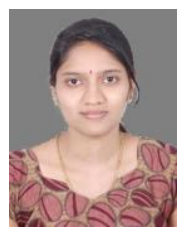

Pooja S, is currently working as an Assistant Professor in Department of Electronics and Communication Engineering at K.S. Institute of Technology, Bangalore. She is currently pursuing her Ph.D at Visvesvaraya Technological University Belgaum and has been awarded B.E. and M.Tech degrees from Visvesvaraya Technological University, Belgaum in 2008 and 2015 respectively. Her current area of research is in Image processing, which has led to various research publications.

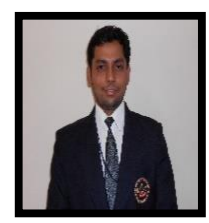

Dr. Mallikarjunaswamy S, is currently working as an Associate Professor in Department of Electronics and Communication Engineering at JSS Academy of Technical Education, Bangalore. He obtained his B.E degree in Telecommunication Engineering from Visvesvaraya Technological University Belgaum in 2008, M. Tech degree from Visvesvaraya Technological University Belgaum in 2010 and was awarded Ph. D from Jain University in 2015.He has 11+ years of teaching experience. His research work has been published in more than 42 International Journals and conference. He received funds from different funding agencies. Currently guiding five research scholars in Visvesvaraya Technological University Belgaum.

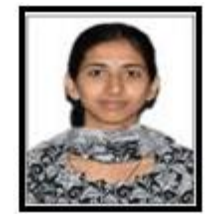

Sharmila N, has completed her B.E in EEE at SJCE, Mysore and M. Tech in CAID at NIE Mysore. Secured second rank in Bachelor of Engineering degree. She has Eight years of experience in teaching and is currently working as an Assistant Professor in RNSIT, Bangalore 